\title{
Note: Combinatorial Alexander Duality-A Short and Elementary Proof
}

\author{
Anders Björner • Martin Tancer
}

Received: 16 October 2007 / Revised: 1 July 2008 / Accepted: 3 July 2008 /

Published online: 14 August 2008

(C) Springer Science+Business Media, LLC 2008

\begin{abstract}
Let $\mathbf{X}$ be a simplicial complex with ground set $V$. Define its Alexander dual as the simplicial complex $\mathbf{X}^{*}=\{\sigma \subseteq V \mid V \backslash \sigma \notin \mathbf{X}\}$. The combinatorial Alexander duality states that the $i$ th reduced homology group of $\mathbf{X}$ is isomorphic to the $\left(|V|-i-3\right.$ )th reduced cohomology group of $\mathbf{X}^{*}$ (over a given commutative ring $R$ ). We give a self-contained proof from first principles accessible to a nonexpert.
\end{abstract}

Keywords Simplicial complex $\cdot$ Homology $\cdot$ Cohomology $\cdot$ Alexander dual

\section{Introduction}

Let $\mathbf{X}$ be a simplicial complex with ground set $V$. For $\sigma \in \mathbf{X}$, let $\bar{\sigma}=V \backslash \sigma$. The Alexander dual of $\mathbf{X}$ is the simplicial complex on the same ground set defined by

$$
\mathbf{X}^{*}=(V,\{\sigma \subseteq V \mid \bar{\sigma} \notin \mathbf{X}\})
$$

See Fig. 1 for an example of a simplicial complex and its Alexander dual.

It is easy to see that $\mathbf{X}^{* *}=\mathbf{X}$. Furthermore, a close homological connection exists between $\mathbf{X}$ and $\mathbf{X}^{*}$, which in the combinatorics folklore has become known as "combinatorial Alexander duality." It states that knowledge of the homology of a simplicial complex gives knowledge of the cohomology of its Alexander dual:

\footnotetext{
A. Björner $(\bowtie)$

Department of Mathematics, Royal Institute of Technology, 10044 Stockholm, Sweden e-mail: bjorner@math.kth.se

M. Tancer

Department of Applied Mathematics, Faculty of Mathematics and Physics, Charles University, Malostranské Náměstí 25, 118 00, Prague, Czech Republic

e-mail: martin@atrey.karlin.mff.cuni.cz
} 
Fig. 1 Simplicial complex $\mathbf{S}$ and its dual

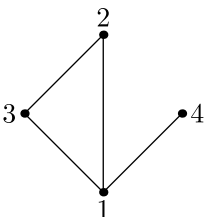

S

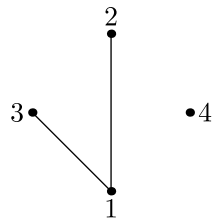

$\mathbf{S}^{*}$

Theorem 1.1 (Combinatorial Alexander Duality) Let $\mathbf{X}$ be a simplicial complex with a ground set of size $n$. Then

$$
\tilde{H}_{i}(\mathbf{X}) \cong \tilde{H}^{n-i-3}\left(\mathbf{X}^{*}\right) .
$$

(Here $\tilde{H}$ stands for reduced homology resp. cohomology over a given ring $R$.)

The earliest explicit statements of Theorem 1.1 that we know of appear in Kalai [10, p. 348] and Stanley [14, p. 184]. Combinatorial Alexander duality is a special case of the original Alexander duality:

Theorem 1.2 (Alexander [1], 1922) Let A be a subset of the sphere $S^{n}$ such that the pair $\left(S^{n}, A\right)$ is triangulable. Then

$$
\tilde{H}_{i}(A) \cong \tilde{H}^{n-i-1}\left(S^{n} \backslash A\right) .
$$

The connection is the following: Suppose that $\mathbf{X}$ is a simplicial complex different from the full simplex with ground set $\{1,2, \ldots, n+2\}$. Let $\mathbf{Y} \cong S^{n}$ be the $n$-skeleton of the full simplex on the set $\{1,2, \ldots, n+2\}$. Let us denote by $\|\mathbf{X}\|$ (resp. $\|\mathbf{Y}\|$ ) the geometric realization of $\mathbf{X}$ (resp. $\mathbf{Y}$ ). Then $\|\mathbf{X}\| \subseteq\|\mathbf{Y}\|$, and it can be shown that $\|\mathbf{Y}\| \backslash\|\mathbf{X}\|$ is homotopy equivalent to $\left\|\mathbf{X}^{*}\right\|$. Thus if $A=\|\mathbf{X}\|$ is seen as a subset of $S^{n}$, we get

$$
\tilde{H}_{i}(\mathbf{X}) \cong \tilde{H}_{i}(A) \cong \tilde{H}^{n-i-1}\left(S^{n} \backslash A\right) \cong \tilde{H}^{(n+2)-i-3}\left(\mathbf{X}^{*}\right)
$$

The Alexander duality theorem has played a very important role in the development of algebraic topology. See [8] or [13] for context and modern treatments and [9] for interesting historical information about James Waddell Alexander, the man and his mathematics.

The modest task of this note is to make the ideas behind combinatorial Alexander duality more widely accessible by giving a "combinatorial" proof from first principles, not relying on more general techniques. It turns out that essentially the same proof was previously given by Dave Bayer [2, 3], see the acknowledgements at the end of the note. Central to our approach is a poset point of view and scrutiny of the combinatorics of the sign labeling of edges in the Boolean lattice $2^{V}$ used for the (co)boundary operations in (co)homology. Applications of combinatorial Alexander duality in combinatorics and algebra can be found for example in [4-7, 10-12, 14-17]. 
Combinatorial Alexander duality exists in more general versions. One such generalization involves induced subcomplexes of $\mathbf{X}$ versus links of faces of $\mathbf{X}^{*}$, see [6, p. 28]. For another one, let $\mathbf{X}$ be a subcomplex of the boundary complex of a $(n-1)$-dimensional convex polytope $P$, and let $\mathbf{X}^{*}$ be the subcomplex of the boundary of the dual polytope $P^{*}$ consisting of faces dual to those faces of $P$ that are not in X. Then Theorem 1.1 still holds, see, e.g., [5] for an application. Furthermore, a very general Alexander duality theorem for nonacyclic Gorenstein complexes appears in $[16$, p. 66]. The reason that we confine this article to the particular simplicial setting of Theorem 1.1, although the idea of the proof is correct in greater generality, is that we want to work with the explicit sign labeling offered by the environment of the Boolean lattice.

\section{Preliminaries}

Let $\mathbf{X}$ be a simplicial complex with ground set $V=\{1,2, \ldots, n\}$. For $j \in \sigma \in \mathbf{X}$, we define the $\operatorname{sign} \operatorname{sgn}(j, \sigma)$ as $(-1)^{i-1}$, where $j$ is the $i$ th smallest element of the set $\sigma$. The following simple property of the sign function will be needed.

Lemma 2.1 Let $k \in \sigma \subseteq\{1,2, \ldots, n\}$ and $p(\sigma)=\prod_{i \in \sigma}(-1)^{i-1}$. Then

$$
\operatorname{sgn}(k, \sigma) p(\sigma \backslash k)=\operatorname{sgn}(k, \bar{\sigma} \cup k) p(\sigma) .
$$

Proof We have that

$$
\operatorname{sgn}(k, \sigma) \operatorname{sgn}(k, \bar{\sigma} \cup k)=\prod_{\substack{i \in \sigma \\ i<k}}(-1) \prod_{\substack{i \in \bar{\sigma} \\ i<k}}(-1)=(-1)^{k-1}
$$

and

$$
p(\sigma) p(\sigma \backslash k)=\prod_{i \in \sigma}(-1)^{i-1} \prod_{i \in \sigma \backslash k}(-1)^{i-1}=(-1)^{k-1} .
$$

In the rest of this section we review the definitions and notation used for (co)homology. Throughout the paper we suppose that $R$ is a commutative ring containing a unit element.

\subsection{Reduced Homology}

Let $C_{i}=C_{i}(\mathbf{X})$ be a free $R$-module with the free basis $\left\{e_{\sigma} \mid \sigma \in \mathbf{X}, \operatorname{dim} \sigma=i\right\}$. The reduced chain complex of $\mathbf{X}$ over $R$ is the complex

$$
\tilde{\mathcal{C}}_{\circledast}(\mathbf{X})=\tilde{\mathcal{C}}_{\circledast}(\mathbf{X} ; R)=\cdots \longleftarrow C_{i-1} \stackrel{\partial_{i}}{\longleftarrow} C_{i} \stackrel{\partial_{i+1}}{\longleftarrow} C_{i+1} \longleftarrow \cdots, \quad i \in \mathbb{Z},
$$

whose mappings $\partial_{i}$ are defined as

$$
\partial_{i}\left(e_{\sigma}\right)=\sum_{j \in \sigma} \operatorname{sgn}(j, \sigma) e_{\sigma \backslash j} .
$$


The complex $\tilde{\mathcal{C}}_{\circledast}(\mathbf{X})$ is formally infinite; however, $C_{i}=0$ for $i<-1$ or $i>\operatorname{dim} \mathbf{X}$. The $n$th reduced homology group of $\mathbf{X}$ over $R$ is defined as

$$
\tilde{H}_{n}(\mathbf{X})=\tilde{H}_{n}(\mathbf{X} ; R)=\operatorname{ker} \partial_{n} / \operatorname{im} \partial_{n+1} .
$$

\subsection{Reduced Cohomology}

Let $C^{i}=C^{i}(\mathbf{X})$ be a free $R$-module with the free basis $\left\{e_{\sigma}^{*} \mid \sigma \in \mathbf{X}, \operatorname{dim} \sigma=i\right\}$. The reduced cochain complex of $\mathbf{X}$ over $R$ is the complex

$$
\tilde{\mathcal{C}}^{\circledast}(\mathbf{X})=\tilde{\mathcal{C}}^{\circledast}(\mathbf{X} ; R)=\cdots \longrightarrow C^{i-1} \stackrel{\partial^{i}}{\longrightarrow} C^{i} \stackrel{\partial^{i+1}}{\longrightarrow} C^{i+1} \longrightarrow \cdots, \quad i \in \mathbb{Z},
$$

where $\partial^{i}=\partial_{i}^{*}$ are maps dual to $\partial_{i}$, explicitly stated:

$$
\partial^{i}\left(e_{\sigma}^{*}\right)=\sum_{\substack{j \notin \sigma \\ \sigma \cup j \in \mathbf{X}}} \operatorname{sgn}(j, \sigma \cup j) e_{\sigma \cup j}^{*} .
$$

The $n$th reduced cohomology group of $\mathbf{X}$ over $R$ is defined as

$$
\tilde{H}^{n}(\mathbf{X})=\tilde{H}^{n}(\mathbf{X} ; R)=\operatorname{ker} \partial^{n+1} / \operatorname{im} \partial^{n} .
$$

\subsection{Relative Homology}

Suppose that $\mathbf{X}$ is a simplicial complex and $\mathbf{A}$ is a subcomplex of $\mathbf{X}$. Let $R_{i}=$ $R_{i}(\mathbf{X}, \mathbf{A})=C_{i}(\mathbf{X}) / C_{i}(\mathbf{A})$, where $C_{i}$ was defined in Sect. 2.1. The relative reduced chain complex of $(\mathbf{X}, \mathbf{A})$ over $R$ is the complex

$$
\tilde{\mathcal{C}}_{\circledast}(\mathbf{X}, \mathbf{A})=\tilde{\mathcal{C}}_{\circledast}(\mathbf{X}, \mathbf{A} ; R)=\cdots \longleftarrow R_{i-1} \stackrel{\mathrm{d}_{i}}{\longleftarrow} R_{i} \stackrel{\mathrm{d}_{i+1}}{\longleftarrow} R_{i+1} \longleftarrow \cdots, \quad i \in \mathbb{Z},
$$

where $\mathrm{d}_{i}$ are defined as

$$
\mathrm{d}_{i}\left(e_{\sigma}+C_{i}(\mathbf{A})\right)=\sum_{j \in \sigma} \operatorname{sgn}(j, \sigma)\left(e_{\sigma \backslash j}+C_{i-1}(\mathbf{A})\right) .
$$

The $n$th relative reduced homology group of $(\mathbf{X}, \mathbf{A})$ over $R$ is defined as

$$
\tilde{H}_{n}(\mathbf{X}, \mathbf{A})=\tilde{H}_{n}(\mathbf{X}, \mathbf{A} ; R)=\operatorname{kerd}_{n} / \operatorname{imd}_{n+1} .
$$

Remark 2.2 When we wish to compute relative homology groups, we can identify $R_{i}=C_{i}(\mathbf{X}) / C_{i}(\mathbf{A})$ with a free $R$-module with the free basis $\left\{e_{\sigma} \mid \sigma \in \mathbf{X}\right.$, $\sigma \notin \mathbf{A}, \operatorname{dim} \sigma=i\}$. Then $\mathrm{d}_{i}$ can be rewritten as

$$
\mathrm{d}_{i}\left(e_{\sigma}\right)=\sum_{\substack{j \in \sigma \\ \sigma \backslash j \notin \mathbf{A}}} \operatorname{sgn}(j, \sigma) e_{\sigma \backslash j} .
$$

One of the important properties of relative homology groups is that they fit into a long exact sequence. See, e.g., [8] or [13] for a proof and more details. 


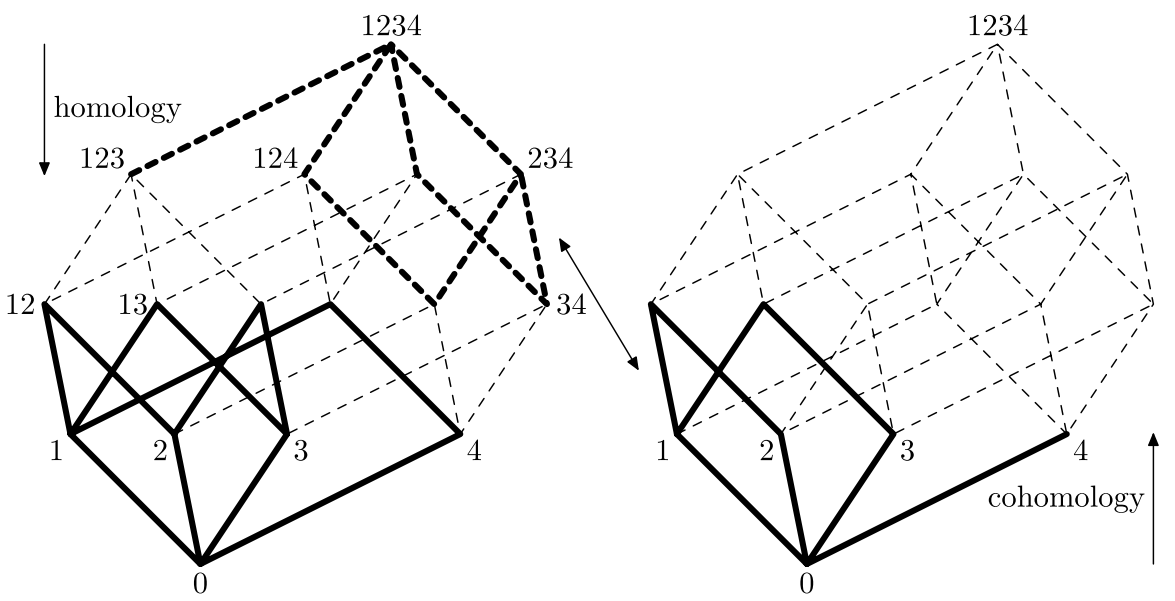

Fig. 2 The lattices $\Gamma_{\mathbf{S}}($ left $)$ and $\Gamma_{\mathbf{S}^{*}}($ right $)$

Lemma 2.3 (Long Exact Sequence of a Pair) Suppose that $\mathbf{X}$ and $\mathbf{A}$ are simplicial complexes $\mathbf{A} \subseteq \mathbf{X}$. Then there is a long exact sequence

$$
\cdots \longrightarrow \tilde{H}_{n}(\mathbf{A}) \longrightarrow \tilde{H}_{n}(\mathbf{X}) \longrightarrow \tilde{H}_{n}(\mathbf{X}, \mathbf{A}) \longrightarrow \tilde{H}_{n-1}(\mathbf{A}) \longrightarrow \cdots
$$

\section{The Idea of the Proof}

Before embarking on the proof of Theorem 1.1, we first present the idea.

Suppose that $\mathbf{X}$ is a simplicial complex with ground set $V$. Let $\Gamma$ be the lattice of all subsets of $V$, and let $\Gamma_{\mathbf{X}}$ be the subposet of $\Gamma$ corresponding to the subsets that are in $\mathbf{X}$. Then the $n$th homology group of $\mathbf{X}$ depends just on the $n$th and $(n+1)$ st levels of the poset $\Gamma_{\mathbf{X}}$. Let $\mathbf{2}^{V}$ be a full simplex with vertex set $V$. It is easy to see that $\tilde{H}_{i}(\mathbf{X}) \cong \tilde{H}_{i+1}\left(\mathbf{2}^{V}, \mathbf{X}\right)$ (see Lemma 4.1). Thus, we restate the problem as computing homologies of the chain complex determined by the complement of $\Gamma_{\mathbf{X}}$-in the sense of Remark 2.2.

The idea of the proof is that if we turn the lattice upside down (exchange $\sigma \subseteq V$ with its complement), then this combinatorial map on generators should induce a canonical isomorphism between the relative homology of the pair $\left(2^{V}, \mathbf{X}\right)$ and the cohomology of $\mathbf{X}^{*}$. This idea is basically correct; however, the mapping is not an isomorphism as described—some sign operations are necessary.

Example 3.1 Let $\mathbf{S}$ be the simplicial complex in Fig. 1. Its ground set is $V_{\mathbf{S}}=$ $\{1,2,3,4\}$. The posets $\Gamma_{\mathbf{S}}$ and $\Gamma_{\mathbf{S}^{*}}$ are depicted in Fig. 2, and the left part of the picture also shows the complement of $\Gamma_{\mathbf{S}}$ (bold, dashed) determining the homology of $\left(2^{V_{s}}, \mathbf{S}\right)$.

In the sense of Remark 2.2, the chain complex $\tilde{\mathcal{C}}_{\circledast}\left(2^{V_{\mathbf{S}}}, \mathbf{S}\right)$ is

$$
\cdots \longleftarrow 0 \longleftarrow\left\langle e_{24}, e_{34}\right\rangle \stackrel{\mathrm{d}_{2}}{\longleftarrow}\left\langle e_{123}, e_{124}, e_{134}, e_{234}\right\rangle \stackrel{\mathrm{d}_{3}}{\longleftarrow}\left\langle e_{1234}\right\rangle \longleftarrow 0 \longleftarrow \cdots,
$$


and the cochain complex $\tilde{\mathcal{C}}^{\circledast}\left(\mathbf{S}^{*}\right)$ is

$$
\cdots \longrightarrow 0 \longrightarrow\left\langle e_{0}^{*}\right\rangle \stackrel{\partial^{0}}{\longrightarrow}\left\langle e_{1}^{*}, e_{2}^{*}, e_{3}^{*}, e_{4}^{*}\right\rangle \stackrel{\partial^{1}}{\longrightarrow}\left\langle e_{12}^{*}, e_{13}^{*}\right\rangle \longrightarrow 0 \longrightarrow \cdots
$$

The map $e_{\sigma} \rightarrow e_{\bar{\sigma}}^{*}$ is not an isomorphism of these two complexes (if $2 r \neq 0$ for $0 \neq r \in R)$, since $\mathrm{d}_{2}\left(e_{234}\right)=-e_{24}+e_{34}$, while $\partial^{1}\left(e_{1}^{*}\right)=-e_{12}^{*}-e_{13}^{*}$. Nevertheless, these two chain complexes are isomorphic, as will be shown in the next section.

\section{The Proof}

The proof of Theorem 1.1 is obtained by combining Lemmas 4.1 and 4.2.

Lemma 4.1 Let $\mathbf{X}$ be a simplicial complex with ground set $V$. Then

$$
\tilde{H}_{i}(\mathbf{X}) \cong \tilde{H}_{i+1}\left(\mathbf{2}^{V}, \mathbf{X}\right) .
$$

Proof This follows from Lemma 2.3. There is the long exact sequence of the pair $\left(2^{V}, \mathbf{X}\right)$ :

$$
\cdots \longrightarrow \tilde{H}_{i+1}\left(\mathbf{2}^{V}\right) \longrightarrow \tilde{H}_{i+1}\left(\mathbf{2}^{V}, \mathbf{X}\right) \longrightarrow \tilde{H}_{i}(\mathbf{X}) \longrightarrow \tilde{H}_{i}\left(\mathbf{2}^{V}\right) \longrightarrow \cdots .
$$

The groups $\tilde{H}_{i+1}\left(\mathbf{2}^{V}\right)$ and $\tilde{H}_{i}\left(\mathbf{2}^{V}\right)$ are zero, hence the groups $\tilde{H}_{i+1}\left(\mathbf{2}^{V}, \mathbf{X}\right)$ and $\tilde{H}_{i}(\mathbf{X})$ are isomorphic.

Lemma 4.2 Let $\mathbf{X}$ be a simplicial complex with ground set $V$ of size $n$. Then

$$
\tilde{H}_{i+1}\left(\mathbf{2}^{V}, \mathbf{X}\right) \cong \tilde{H}^{n-i-3}\left(\mathbf{X}^{*}\right) .
$$

Proof Suppose that $V=\{1,2, \ldots, n\}$. The chain complex for reduced homology of the pair $\left(\mathbf{2}^{V}, \mathbf{X}\right)$ is the complex

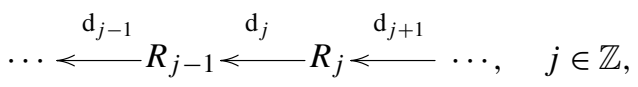

where $R_{j}=\left\langle e_{\sigma} \mid \sigma \subseteq V, \sigma \notin \mathbf{X}, \operatorname{dim} \sigma=j\right\rangle$, and $\mathrm{d}_{j}$ are the unique homomorphisms satisfying

$$
\mathrm{d}_{j}\left(e_{\sigma}\right)=\sum_{\substack{k \in \sigma \\ \sigma \backslash k \notin \mathbf{X}}} \operatorname{sgn}(k, \sigma) e_{\sigma \backslash k} .
$$

The cochain complex for reduced cohomology of $\mathbf{X}^{*}$ is the complex

$$
\cdots \stackrel{\partial^{j-1}}{\longrightarrow} C^{j-1} \stackrel{\partial^{j}}{\longrightarrow} C^{j} \stackrel{\partial^{j+1}}{\longrightarrow} \cdots, \quad j \in \mathbb{Z},
$$


where $C^{j}=\left\langle e_{\sigma}^{*} \mid \sigma \subseteq V, \operatorname{dim} \sigma=j, \sigma \in \mathbf{X}^{*}\right\rangle=\left\langle e_{\sigma}^{*}\right| \sigma \subseteq V, \operatorname{dim} \bar{\sigma}=n-j-2$, $\bar{\sigma} \notin \mathbf{X}\rangle$, and $\partial^{j}$ are the unique homomorphisms satisfying

$$
\partial^{j}\left(e_{\sigma}^{*}\right)=\sum_{\substack{k \notin \sigma \\ k \cup \sigma \in \mathbf{X}^{*}}} \operatorname{sgn}(k, \sigma \cup k) e_{\sigma \cup k}^{*}=\sum_{\substack{k \in \bar{\sigma} \\ \sigma \backslash k \notin \mathbf{X}}} \operatorname{sgn}(k, \sigma \cup k) e_{\overline{\bar{\sigma} \backslash k}}^{*} .
$$

Define $p(\sigma)$ as in Lemma 2.1 and let $\phi_{j}: R_{j} \rightarrow C^{n-j-2}$ be the isomorphisms generated by the formula

$$
\phi_{j}\left(e_{\sigma}\right)=p(\sigma) e_{\bar{\sigma}}^{*}
$$

for $\sigma \notin \mathbf{X}$ with $\operatorname{dim} \sigma=j$ (note that these two conditions are equivalent to $\operatorname{dim} \bar{\sigma}=$ $\left.n-j-2, \bar{\sigma} \in \mathbf{X}^{*}\right)$. We then have the diagram

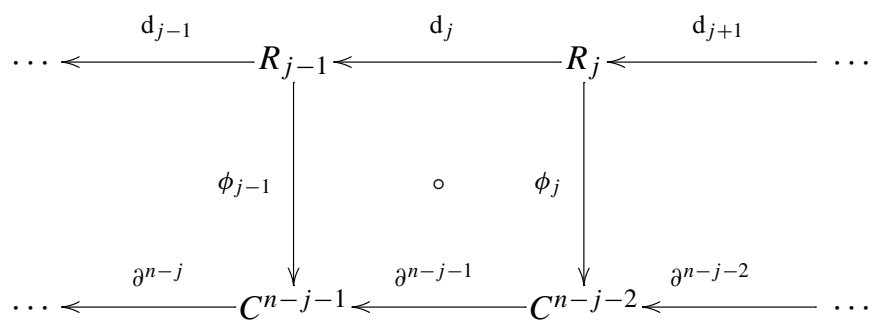

We check that $\phi_{j-1} \circ \mathrm{d}_{j}=\partial^{n-j-1} \circ \phi_{j}$. Let $\sigma \subseteq V, \sigma \notin \mathbf{X}, \operatorname{dim} \sigma=j$. Then

$$
\begin{gathered}
\phi_{j-1} \circ \mathrm{d}_{j}\left(e_{\sigma}\right)=\phi_{j-1}\left(\sum_{\substack{k \in \sigma \\
\sigma \backslash k \notin \mathbf{X}}} \operatorname{sgn}(k, \sigma) e_{\sigma \backslash k}\right)=\sum_{\substack{k \in \sigma \\
\sigma \backslash k \notin \mathbf{X}}} \operatorname{sgn}(k, \sigma) p(\sigma \backslash k) e_{\frac{\sigma}{\sigma k}}^{*}, \\
\partial^{n-j-1} \circ \phi_{j}\left(e_{\sigma}\right)=\partial^{n-j-1}\left(p(\sigma) e_{\bar{\sigma}}^{*}\right)=\sum_{\substack{k \in \sigma \\
\sigma \backslash k \notin \mathbf{X}}} p(\sigma) \operatorname{sgn}(k, \bar{\sigma} \cup k) e_{\frac{\sigma \backslash k}{}}^{*}
\end{gathered}
$$

These two sums are equal term by term, due to Lemma 2.1. Thus $\phi$ is an isomorphism of the complexes, implying

$$
\tilde{H}_{i+1}\left(\mathbf{2}^{V}, \mathbf{X}\right) \cong \tilde{H}^{n-i-3}\left(\mathbf{X}^{*}\right) .
$$

Acknowledgements We are grateful to Jiri Matousek and Kathrin Vorwerk for helpful remarks.

An anonymous referee has pointed out to us that the proof presented in this note is essentially the same as one previously given by Dave Bayer, see the appendix to his unpublished notes [2] and the remark after Theorem 2.1 in [3].

\section{References}

1. Alexander, J.W.: A proof and extension of the Jordan-Brouwer separation theorem. Trans. Am. Math. Soc. 23, 333-349 (1922)

2. Bayer, D.: Monomial ideals and duality. Lecture notes (1996). http://www.math.columbia.edu/ bayer/ papers/Duality_B96/ 
3. Bayer, D., Charalambous, H., Popescu, S.: Extremal Betti numbers and applications to monomial ideals. J. Algebra 221, 497-512 (1999)

4. Björner, A., Butler, L.M., Matveev, A.O.: Note on a combinatorial application of Alexander duality. J. Combin. Theory Ser. A 80, 163-165 (1997)

5. Björner, A., Ziegler, G.M.: Combinatorial stratification of complex arrangements. J. Am. Math. Soc. 5, 105-149 (1992)

6. Buchstaber, V.M., Panov, T.E.: Torus Actions and Their Applications in Topology and Combinatorics. Univ. Lecture Series, vol. 24. Am. Math. Soc., Providence (2002)

7. Eagon, J.A., Reiner, V.: Resolutions of Stanley-Reisner rings and Alexander duality. J. Pure Appl. Algebra 130, 265-275 (1998)

8. Hatcher, A.: Algebraic Topology. Cambridge University Press, Cambridge (2001)

9. James, I.M.: Portrait of Alexander (1888-1971). Bull. Am. Math. Soc. 38, 123-129 (2001)

10. Kalai, G.: Enumeration of $Q$-acyclic simplicial complexes. Israel J. Math. 45, 337-351 (1983)

11. Kalai, G., Meshulam, R.: A topological colorful Helly theorem. Adv. Math. 191, 305-311 (2005)

12. Miller, E., Sturmfels, B.: Combinatorial Commutative Algebra. Springer, New York (2005)

13. Munkres, J.R.: Elements of Algebraic Topology. Addison-Wesley, New York (1984)

14. Stanley, R.P.: Linear Diophantine equations and local cohomology. Invent. Math. 68, 175-193 (1982)

15. Stanley, R.P.: Some aspects of groups acting on finite posets. J. Combin. Theory Ser. A 32, 132-161 (1982)

16. Stanley, R.P.: Combinatorics and Commutative Algebra, 2nd edn. Birkhäuser, Boston (1996)

17. Stanley, R.P.: Enumerative Combinatorics, vol. 1. Wadsworth \& Brooks/Cole, Monterey (1986). Reissued by Cambridge Univ. Press, Cambridge (1997) 